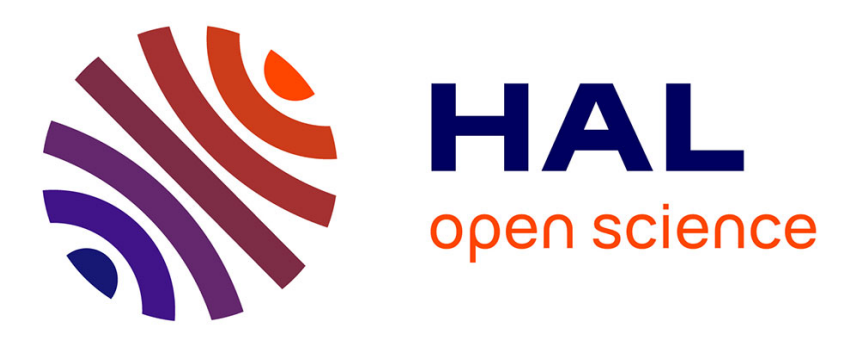

\title{
Synchronisation and desynchronisation as important elements for the development of interaction capabilities
}

Eva Ansermin, Philippe Gaussier, Antoine de Rengervé, Ghiles Mostafaoui, Ludovic Marin

\section{- To cite this version:}

Eva Ansermin, Philippe Gaussier, Antoine de Rengervé, Ghiles Mostafaoui, Ludovic Marin. Synchronisation and desynchronisation as important elements for the development of interaction capabilities. IEEE ICDL-EPIROB, Sep 2017, Lisboa, Portugal. hal-01593325

\section{HAL Id: hal-01593325 \\ https://hal.science/hal-01593325}

Submitted on 26 Sep 2017

HAL is a multi-disciplinary open access archive for the deposit and dissemination of scientific research documents, whether they are published or not. The documents may come from teaching and research institutions in France or abroad, or from public or private research centers.
L'archive ouverte pluridisciplinaire HAL, est destinée au dépôt et à la diffusion de documents scientifiques de niveau recherche, publiés ou non, émanant des établissements d'enseignement et de recherche français ou étrangers, des laboratoires publics ou privés. 


\title{
Synchronisation and desynchronisation as important elements for the development of interaction capabilities
}

\author{
Eva Ansermin ${ }^{1}$, Philippe Gaussier ${ }^{1}$, Antoine de Rengervé ${ }^{2}$, Ghiles Mostafaoui ${ }^{1}$ and Ludovic Marin ${ }^{3}$
}

\begin{abstract}
As non verbal cues are crucial for communication hence long-life learning. Here we focus on the advantages of using rhythmic feedback as implicit marker during interactions. Through two different experimental studies with naive subjects and robots we show the utility of using and modelling rhythmic adaptation but also desynchronisation. We found out that rhythmical adaptation and bidirectional entrainment are imperative to reach synchronisation. Yet, we discovered that desynchronisation can be perceived as an implicit marker and hence be used to predict an issue in the interaction, making this interaction more pleasant. This shows that rhythmic cues such as synchronisation and desynchronisation are important elements for the development of interaction capabilities.
\end{abstract}

Index Terms - Synchronisation, Entrainment effect, bidirectional interactions, non verbal communication, interaction capabilities.

\section{INTRODUCTION}

In this paper we focus on the nonverbal information contained in the rhythmic stimuli, especially the phenomenon of unintentional synchronisation. Humans are experts in analysing (sometimes unintentionally) a context of environmental information and the motion of their partner to achieve a cooperative task [1]. Moreover, the absence of those significant cues (such as coordination, synchronisation, imitation, joint attention, etc.) can impact the efficiency of a task. Synchronisation occurs when two signals are at the same frequency and in phase or in opposition of phase and is very present in nature (birds in flight, fireflies twinkling, pendulum etc. [2]). It is present between humans as well as it can be observed in daily examples when we synchronize our pace or arm movements. Humans tend to reach unintentional synchronisation when walking or performing a task together. In general terms, a human facing a rhythmical stimuli (visual, audio or even tactile) will tend to synchronize. This phenomenon is spontaneous but is also inevitable even when subjects try not to be rhythmically influenced [3]. However, synchronisation is not necessarily reached when facing a rhythmical stimulus, especially if the proper (natural) frequency of the subject is too far from the frequency of this external signal. Yet, what can be observed is that the human motion is nevertheless influenced and its frequency is closer to the stimuli's [4]. This phenomenon is called the entrainment effect or magnet effect and can be seen as the source of synchronisation. In concrete terms,

This research was supported by the DGA, the Agence Nationale de la Recherche grant (DIRAC Project ANR 13-ASTR-00188-01) and Robotex

${ }^{1}$ ENSEA, University of Cergy-Pontoise, UMR CNRS 8051 ETIS, FRANCE. ${ }^{2}$ Partenering Robotics ${ }^{3}$ EuroMov laboratory, Univ. Montpellier, Montpellier, France synchronisation between humans could be a stable state between two systems which entrain each other. Actually, it is admitted, in the literature, that subjects can be unintentionally synchronized when the external rhythmical stimuli is around $10 \%$ of their own proper frequency (also called natural frequency or eigen-frequency). Unintentional synchrony is very important in our rhythmic interaction and plays a key role in coordination [4] but also in social interaction in general. Indeed, numerous research have put forward the link between attention to our interaction partner and synchronisation with this same partner [5] [6] [7]. In general a lot of works have shown the positive emotional effect of synchronisation in communication (better learning with a teacher [8], interaction of the mother with her baby [9] etc.). Such phenomenon have also be reported during HRI. For example, Ogawa et al. reported an entrainment of nodding between a human and a robot [10] and Sakamaoto et al showed a person's body movements are entrained to robot's ones in the situation of a route guide[11].

As an important nonverbal cue, using rhythmical feedback and synchronisation seems very important in the frame of long life learning. Indeed, as synchronisation permits to ease attention and concentration [12] [4], being able to synchronize is real advantage to enhance efficiency during joint tasks. Actually, synchronisation has already been used as an implicit marker to make the robot focus on a plausible interacting partner [13]. However, it was proven by Lorentz et al. the human have more difficulty to unintentionally synchronize with a robot that is not rhythmically adaptive [14].On the contrary, nonsynchronized rhythm can be disturbing during a task [15] and even negative in a psychological approach as shown by Lumsden et al. [16]. Thus an interruption of synchronisation and a variation of rhythm during an interaction could constitute an implicit negative marker that could be exploited.

In more general term, we argue that rhythmic feedback such as synchronisation are vital not only during the interaction but also for the development of interaction capacities. In a previous paper, we showed that imitation could be a way to exhibit learning and communication capabilities [17] but also enhance interaction with autistic children [18]. We also put forward that synchronisation could be used as an internal reward for learning [19].

In this paper, we put forward two experimental studies to highlight the consequences of entrainment, synchronisation and desynchronisation in a interaction, especially in term of implicit markers and communication abilities. First, we will study the condition to reach unintentional synchronisation with a robot during rhythmical interaction between a NAO robot and a human. For this purpose we propose a neural model of entrainment that will be tested on naive 
subjects for different movements. We will study the advantages of implementing such model versus using a non-adaptive moving robot. Our second experiment deals with the use of desynchronisation during a navigation task between a human and another robot. We will study how can rhythm feedback influence the perception of the human and be used for predicting an issue in the interaction.

\section{ENTRAINMENT EFFECT MODEL}

As stated before, studying unintentional synchronisation and rhythmic entrainment implies first making the infant or the robot able to be rhythmically entrained by the parter' $s$ motion dynamics. Our approach is based on dynamical systems theories. In such approaches, we model the agents with oscillators whose dynamics influence and entrain each other (as Hugen's pendulums). As a simple example, two pendulums (at different frequencies) on the same table can synchronize because of the vibration of this table which permit an energy exchange between the two systems [20]. Pantaleone linked those mechanical synchronisation to biological ones [20]. Haken Kelso and Bunz proposed a system of equations modelling the behavior of coupled Van Der Pol oscillators [21]. By using dynamical systems, we consider that agents bring energy to each other via stimuli (visual for example) to entrain the partner's rhythmic behavior in a very low level process and consequently change their mutual frequency and motion amplitude.

Modelling two systems (agents) rhythmically influencing each other using dynamical systems implies the use of coupled oscillators, whether they are mathematical [21] or neural [22]. In our case we used a neural model with an entrainment effect model we recently proposed [23] (see section 1). This model has been validated with naive subjects and has been proven to be efficient to adapt the robot's rhythm to the human motion dynamics. However, it has not been tested on different moves and tasks.

For clarity sake, this model will be explained briefly below but we invite the reader to refer to [23] for more details.

The model uses a neural oscillator to control the robot rhythmical movement whose dynamic can be influenced by an energy coming from the optical flow induced by the human motion. The global model is illustrated figure 1 .

Figure 1 shows a neural oscillator inspired by the Prepin model [24]. The equation of this oscillator according to the parameters shown in figure 1 is given by equation 1 and 2 ;

$$
\begin{aligned}
& N_{1}(n+1)=N_{1}(n)-\beta N_{2}(n)+\alpha 1 \\
& N_{2}(n+1)=N_{1}(n)+\beta N_{2}(n)+\alpha 2
\end{aligned}
$$

The output of both neurons is consequently oscillatory and the frequency depends on the parameter $\beta$.

This oscillator controls the robot's arm and can be influenced by the movement of an interacting partner. A camera is located in front of the robot. The optical flow is extracted from the images by a hierarchical algorithm proposed by Amiaz et al. [25]. The optical flow is then used to obtain directional neurons sensitive to vertical or horizontal movements. When the movement is rhythmic, these neurons provide a periodical response which can alternate between negative and positive values

$$
N 1(n+1)=N 1(n)-\beta N 2(n)-\alpha 1+c p * f(n)
$$

This signal is then modulated by a coupling factor $c p$ and added to the oscillator signal (see equation 3). This added signal changes the dynamic of the oscillator and can lead to synchronisation with the partner. The coupling factor $c p$ allows us to weight the amplitude of the input signal and so the range of frequencies in which the oscillator would be able to be synchronized; if the coupling factor is high, the oscillator will be able to synchronized in a large range of frequencies and vice versa. The figure 2 shows the simulated resulting entrainment of the oscillator for two different coupling factors. We can observe that, for a small coupling factor (0.05), the oscillator is influenced and its dynamic is deformed but is not at the same frequency as the simulated optical flow induced by human motion. On the contrary we can see that a higher coupling factor (0.2) allows the oscillator to have the same frequency as the simulated optical flow signal. Precisely, we can see that those two signals are out of phase with a shift of $\pi / 2$. The optical flow signal being the derivative of the human arm position, this means that the oscillator is indeed synchronized with the human arm movement.

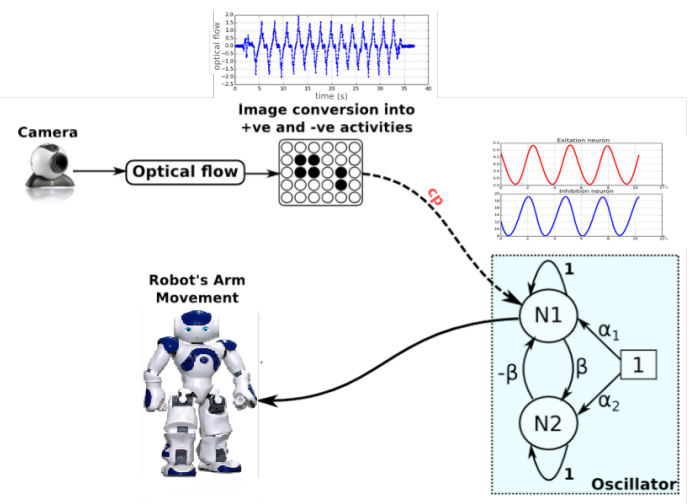

Fig. 1: The entrainment model used during the experiments

\section{Studying UNINTENTIONAL RHYTHMiC ENTRAINMENT IN HRI}

We performed experiments for studying the visual entrainment effect in HRI during imitation games between a NAO robot and naive subjects. Two main types of interaction were analysed : i) unidirectional : the robot moves with a fixed frequency of $0.73 \mathrm{~Hz}$ without taking into account the human motion dynamic ii) bidirectional : the robot is able to be adaptive to the human rhythm using the previously presented neural model. We compared the two conditions to highlight the importance of bi-directionality during rhythmical interaction. This part sums up our result. For more detail about this experiment please see [26].

\section{A. Experimental set up}

Each participant stands facing the NAO robot about 1.5 meter in distance. The robot was seated on a small chair on a table and at the same height as the human partner (see figure 3). The robot can perceive 

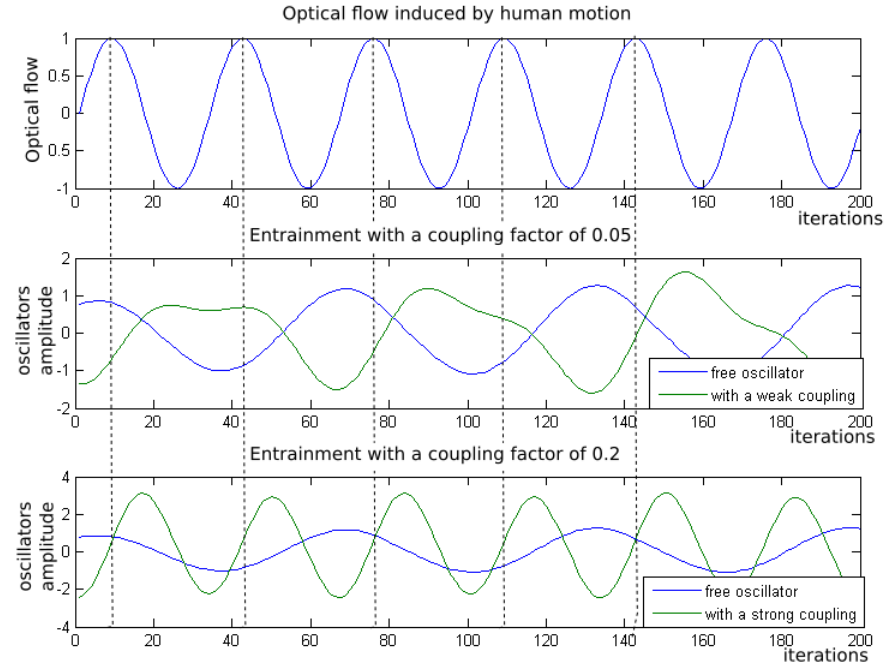

Fig. 2: Results on the Same rhythmic entrainment of the oscillator for two different coupling factors

the human partner through an external camera to avoid the limitations of the NAO's one. The collected data (the oscillator controlling the robots arm and the optical flow induced by the human movements) are saved directly during the experiments.

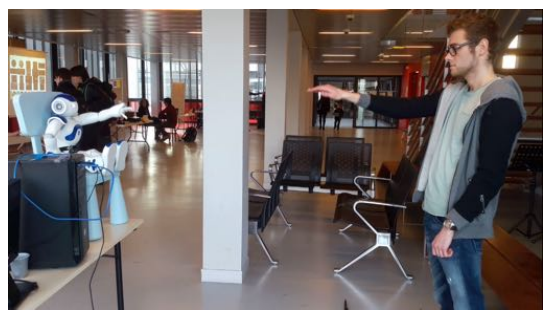

Fig. 3: Experimental set up

1) Subjects: The experiments were conducted on nine young healthy adults. Two women and seven males (average age: 25 years old, all students).

2) Condition: For studying the human unintentional entrainment property, the real goal of the experiments was hidden from the participants, they were told that the objective was to build a data base for teaching the robot to recognize different gestures. It is important to note that they were explicitly asked to move naturally regardless to the robot behavior.

We studied three rhythmical movements:

- (1) raising and lowering the arms vertically (mov1)

- (2) raising and lowering the arm horizontally(mov2)

- (3) waving at the human (mov3)

Video showing NAO producing those movements and a practical example of the entrainment model are available at this link 1 .

Each subject performed the three movements in three different conditions, always in the same order.

- First condition: the purpose of this part was to obtain the proper frequency (natural frequency)

${ }^{1}$ www.etis.ensea.fr/neurocyber/Videos/authors/ansermin/icdl2017 of the participants for each movement. We explained the subjects the movement and ask them to do it alone at their own preferred rhythm. To ensure the spontaneity of the movement we maintained conversation with the subject. Each movement was done for 30 seconds in a random order.

- Second condition: here, we studied the influence of the robot which moved at its own pace (without entrainment or adaptation). Consequently we studied the entrainment effect in an unidirectional context. The robot was just an automaton and was not influenced by the rhythm of the human. We asked the subject to do the same movement as the robot but still at their own pace as in the first condition. The robot's purpose was just to show them which movement is to be done. The three movements were made in a random order, each during 30 seconds.

- Third condition: this part concerned the study of entrainment effect in a bidirectional interaction. The robot was here able to be entrained by the partner thanks to the model presented in the previous section. We fixed the coupling factor to a value of 0.1 so that the robot would be able to synchronise around $15 \%$ (maximum 20\%) of its own frequency. Like previously, the subject was asked to do the same movement as the robot at their own rhythm and the three movements were ordered randomly.

3) Hypothesis: We expected to obtain, in the second condition, an entrainment effect on the human movement and eventually synchronisations if the robot and the partner's preferred frequency are closed enough (around $10 \%$ if we consider the data obtained for HHI in the literature). For the third condition, we expected to find a large majority of cases where the robot and the humans are synchronized which would occur, due to the mutual rhythmic entrainment, at a frequency between the robot's original one $(0.73 \mathrm{~Hz})$ and the human's preferred frequency (given by the first condition).

\section{B. Results}

The comparison of frequencies during the three tasks are summed up in figure 4 where the frequencies are detailed for each movement. Gaps are due to missing datas or issue during the subject's passage with the subject or the recording.

1) Unidirectional entrainment: As can be observed in figure 4, every participant's frequency was modified between condition 1 and 2. Moreover, frequency during condition 2 was always closer to the robot's proper frequency, putting forward the influence of the moving robot on the human: the entrainment effect. We even found case of synchronisation (9 precisely). We can take note that this usually corresponds to the subjects having proper frequencies (in condition 1) close to the robot's one $(0.73 \mathrm{~Hz})$. In particular, the four cases where the subject proper frequency was in a range of $10 \%$ from the robot were all synchronized during the second condition which is in the line of the results obtained for unintentional synchronisation in Human Human Interactions.

However we found out that the gap between the subjects' natural frequencies and their frequencies during condition 2 was in average $23 \%$ and go from from $4 \%$ to $50 \%$ difference. This mean is clearly higher 


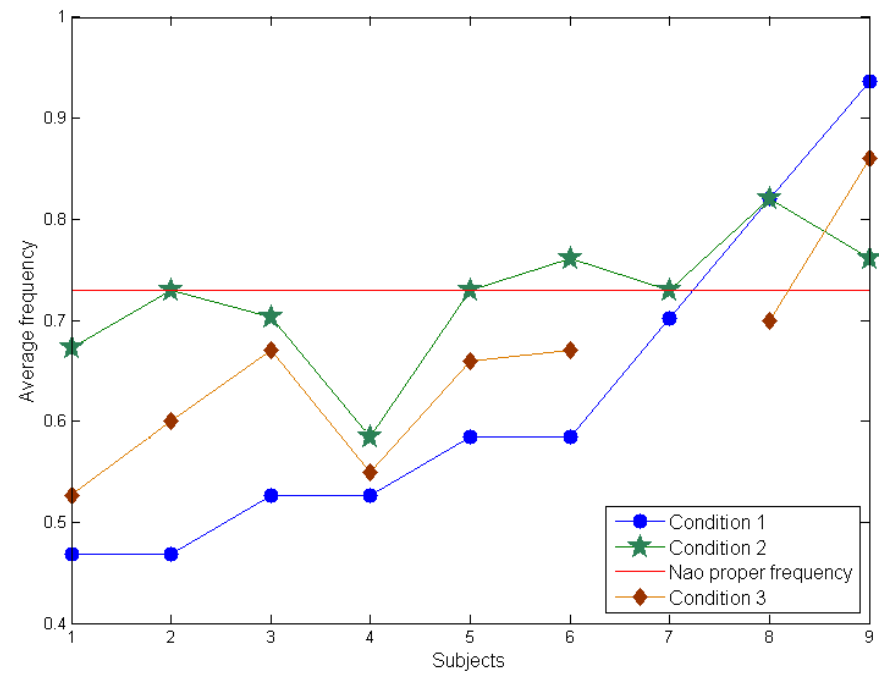

(a) Frequencies during the three conditions for mov1

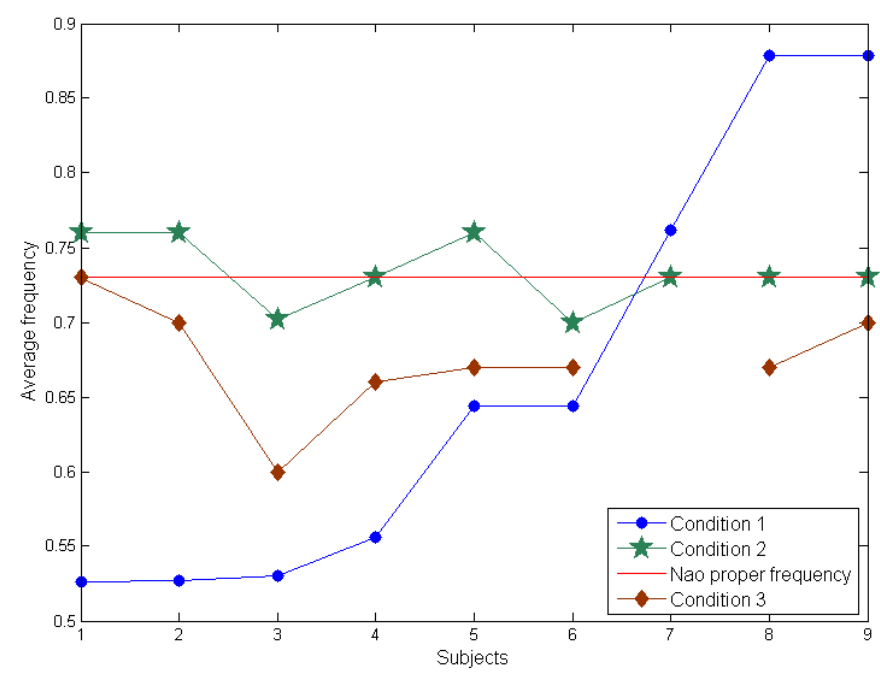

(b) Frequencies during the three conditions for mov2

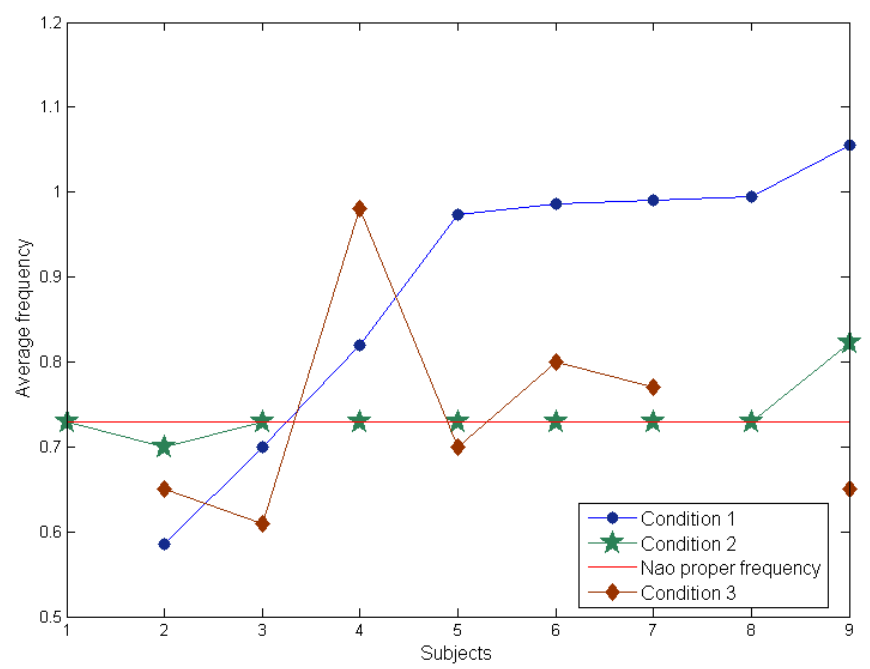

(c) Frequencies during the three conditions for mov3

Fig. 4: Average motion frequencies while performing the three interactive movements during at the three different conditions for all the movement than what we expected (around $10 \%$ in HHI). We actually wondered what were the cause of such difference. We argue that this could be because we lacked a covering task to completely ensure the unintentional part of the process. Another interesting result is that the entrainment is not the same according to the movement we consider; indeed if we consider only the synchronized cases during this second condition, we notice that the third movement (mov3) is the one where we can find the most case of synchrony. Considering only the cases of synchrony, it is the movement corresponding to the most important entrainment $19 \%$. We wonder if this is the case because this movement (waving, saying hello) is particularly social.

We put forward the fact that using only a moving robot wasn't enough to reach synchrony in a majority of cases as it was indeed highlighted in the state of art.

2) Bidirectional entrainment: During this condition, we used the previously detailed entrainment model, the robot was here able to be adaptive and could be influenced by the subject. In this case, $100 \%$ of the participants were synchronized with the robot. We note that $75 \%$ of the interaction frequencies were located between the own frequency of the robot and the subject ones; the rhythmic adaptation came from both agents. This can be observed in figure 4 where we can clearly see that the frequency of the third condition is located between the two other curves (conditions 1 and 2). This means that the human was influenced as well as the robot.

However, the entrainment is less important thanks to the robot's on adaptation. In average, we have an entrainment in average of $19 \%$ and an important number of cases around $15 \%$. This condition shows the symmetric role of the human and the robot and the necessity for both side to be entrained to reach synchronization. Despise the difference between the proper frequency of all our subject, we were able to get a synchronisation for every movement in every case thank not only to the robot adaptation but to the human 's rhythmical adaptation as well. In our cases bilateral entrainment is therefore necessary to reach synchronisation. As shown by the state of art this synchronisation could be one of the keys to a better interaction with robots.

\section{DESYNChronisAtion AS A NEGATIVE MARKER}

This experiment aims at studying desynchronisation. Especially we wonder if this rhythmical feedback can be used as an implicit communication mechanism to signal a failure during a task involving two interactive agents (a typical situation for online teaching).

\section{A. Experimental set up}

During those experiments our cover story was a navigation task in which the subject had to teach an indoor round to a robot. We used the robot DIYA ONE (cf figure5) which has been designed by the Partnering robotics. Subjects had to walk alongside the robot during each section and were given a tablet to control the robot (speed and rotation) which was supposed to learned the way. Pictures of a subject teaching the robot and the round map can be seen in figure 6.

During this rounds, the robot emitted a regular beeping sound that was controlled by our neural 


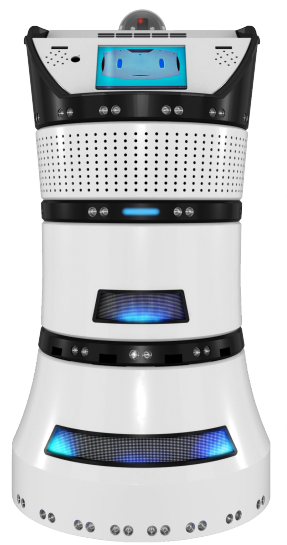

Fig. 5: the DIYA ONE robot designed by Partnering robotics

oscillator without external feedback, hence a fixed frequency. This sound constituted our rhythm feedback to entrain the human walking pace. The frequency of this sound was always closed enough to the subject pace frequency to make the subject synchronise with DIYA ONE during the round, see figure 7 for a practical example. Thank to this entrainment (rhythmic sound), the human's pace and beeping sound were at the same frequency and synchronised (one step for each beep) during the rounds. During some rounds a very brief desynchronisation of the beeping sound preceded "accidents" that were programmed on the way. We studied the influence of this desynchronisation on the behaviour of the subject.

1) Subjects: The experiment was conducted on 11 males subjects including 10 students and 1 professor with a average age of 27 year old.

2) Condition: We explained the subject that the robot has learned the round but that this learning was incomplete. Their task was to test this learning and, if necessary, to correct the trajectory with the tablet to improve the learning. The subject had to perform two rounds which he believed were conducted with two different neural networks controlling the robot. Their official purpose was therefore to evaluate both networks.

In reality, both network were the same and the robot's teaching of the round was already well done. However, we added two fake 'accidents' on each round. We decided of two accidents position for the first trial and two other accident positions in the second trial (see figure 6). The two rounds were separated as such:

- Condition (1) : During this round, the robot emitted a regular beeping sound and kept the same frequency during the whole turn.

- Condition 2 (2): During this round, the sound frequency was fixed as well but doubled during the period before the accident (see figure 8)

Condition 1 and 2 were alternated in a random way. The experimenter discussing with the subject did not know which experiment was selected.

After the rounds, the participant was invited to fill out a questionnaire of three items:

- To rank the ease of the robot control (from 0 to 5)

- To rank the quality of walking with the robot (from 0 to 5)

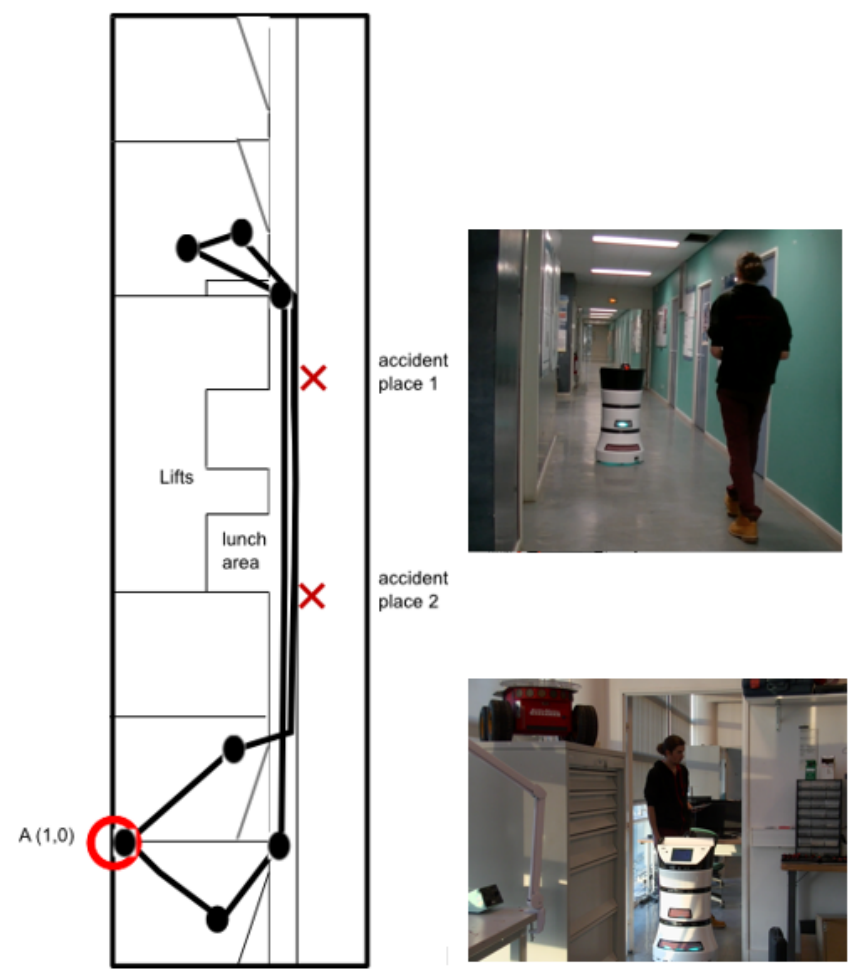

Fig. 6: The round map (start at the indication $\mathrm{A}(1,0)$ ) and two pictures of a subject walking behind DIYA ONE during the task.

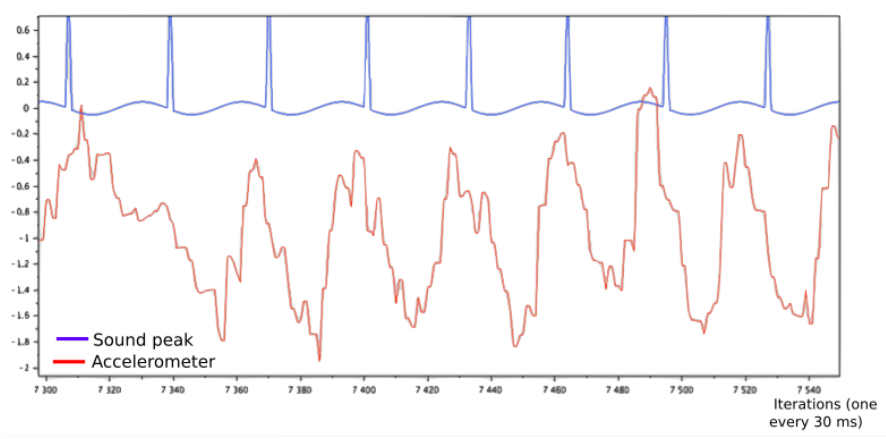

Fig. 7: In blue, the merging of the oscillator output and the triggering of sound (the peaks). The sound is emitted on the first zero crossing of the oscillator. In red the data measured with the accelerometer on the tablet (value centred according to the mean value during the experiment and scaled). These values are directly related to the walking pace and the swinging of the arms (the subject holds the tablet and its sensors in his/her hands). We can observed that the two signals are at the same frequency. 


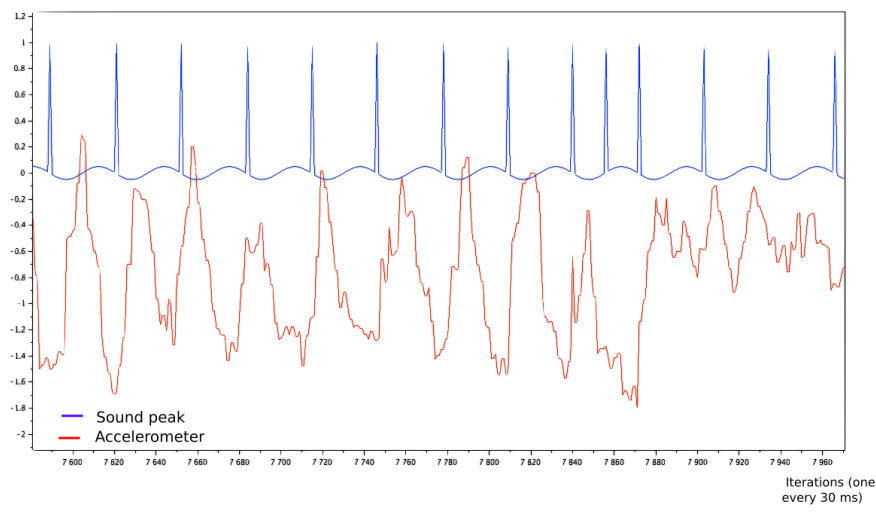

Fig. 8: In blue, rhythmic sound emission and one sound proceeding an "accident" triggered by pone of the experimenters. In red the data measured with the accelerometer on the tablet.

- What network (condition) they preferred.

They were also invited to note down every remark or details they noticed.

It is important to note that some accidents also occurred without being programmed due to a mistake of the human partner or to a fail in the network system. In those case, they were no desynchronisation in the beeping sound.

3) Hypothesis: Because the beeping modification was very discrete (change of the frequency during one period only), we expected to have a small preference and better mark in favour of condition 2 .

\section{B. Result}

The result of the ranking is detailed in a chart figure 9 according to the condition but also according to the order of the condition. Indeed, we can observed that the second condition is always perceived as easier in term of robot control. This is of course due to the fact that the subject is more used to the robot in the second trial.

Concerning the ranking by condition, we notice that, contrary to our predictions, the ranking is not better for condition 2 . The robot control is slightly better for condition 1 . This small difference can also be a consequence of the random order of the trial. Walking with the robot is also consider more agreeable for condition 1 . However, we can notice that the standard deviation is less important for condition 2.

Changing the rhythm before an accident seems to have no effect at all if we consider the ranking. Yet, now if we ask people which one they preferred the results are clearly not ambiguous. Figure 10 shows the result for this question. This result is really conclusive as $100 \%$ of the participants preferred the condition with the changing rhythmical feedback. This was even the case when they reported more accidents (related to true robot accidents) during this condition.

What is very surprising is that subjects actually ranked this condition more severely than the other but still chose to prefer it. Beside, according to the questionnaire, no one noticed the variations in the rhythm before the accident. They were indeed no remark about the beeping sound in the comparison between the two conditions reported by the subjects despite that it was actually the only difference. This

\begin{tabular}{|c|c|c|c|c|}
\cline { 2 - 5 } \multicolumn{1}{c|}{} & $\begin{array}{c}\text { robot } \\
\text { control }\end{array}$ & sd & walking with robot & sd \\
\hline Condition 1 & 3,75 & 0,99 & 4,13 & 0,80 \\
\hline Condition 2 & 3,65 & 0,67 & 3,65 & 0,67 \\
\hline & 3,55 & 0,96 & 4,23 & 0,68 \\
\hline $\begin{array}{c}\text { First trial } \\
\text { (random } \\
\text { condition) }\end{array}$ & & & & \multicolumn{2}{c|}{} \\
\hline $\begin{array}{c}\text { Second trial } \\
\text { (random } \\
\text { condition) }\end{array}$ & 3,86 & 0.71 & 4,23 & 0,75 \\
\hline
\end{tabular}

Fig. 9: Average and standard deviation of the ranking of the robot. This is detailed by condition but also according to the order of the condition.

\begin{tabular}{|c|c|}
\cline { 2 - 2 } \multicolumn{1}{c|}{} & preference \\
\hline Condition 1 & 0 \\
\hline Condition 2 & 11 \\
\hline
\end{tabular}

Fig. 10: Preference of the subject between the two conditions (condition 2: desynchronisation before accident)

means that, as we thought, this change was too discreet to be perceived consciously but still played a role on the subject' s feeling. This role was remarkable as it concerned every participants.

This is a major result as the impact of rhythm variation is clearly significant (100\% of the cases) but is also unnoticed by the subjects. We can conclude that participants saw an implicit negative marker in the desynchronisation of the robot. This allowed them to anticipate accidents or in more general terms, trigger the interruption of the interaction.

\section{Conclusion and Discussion}

In this paper, we presented two experimental studies concerning the rhythmical feedback of a robot. The first one put forward the interest modelling the entrainment effect to reach synchronisation which is a positive implicit marker in interaction. The second one, on the contrary, studied how can a change of rhythm, a desynchronisation, between a human and a robot be exploited as a negative implicit marker.

As shown by the state of art, synchronisation is an important implicit marker in HHI. The study with a NAO robot shows that unintentional rhythmic entrainment and synchronisation can be present during HRI. The human is unintentionally entrained by the moving robot. Yet, this unidirectional entrainment is not enough to reach a stable synchronous state. Only the addition of such a rhythmical feedback launches synchronisation. This highlights that bilaterality is very important when it comes to coordination or synchronisation.

Our second experiment shows that a brutal change in a rhythm during a HRI could also be perceived as a non verbal cue by the human. $100 \%$ of our subjects find more pleasant to control a robot with such a rhythmical feedback. A very interesting result is the 
unintentional aspect of this preference. We defend that this rhythmical feedback allows the subject to anticipate an issue in the interaction and thus constitutes a reward. The way this signal was processed and used should be investigated in future experiments.

In general term, we showed that rhythmical feedbacks (whether it is an adaptation or a desynchronisation) could have an impact on the quality of the interaction during dyadic interaction (parent-infant for instance and more generally long life learning). Of course, those signals could also be more explicit and "classical". For example, a agent could inform its partner of a future accident by emitting a single beeping. Yet, we argue such explicit cues are a heavier cognitive load for the subject. Using implicit, unconscious feedback allows the human to stay focus on his main task and ease the concentration. Here we showed that exploiting rhythm feedback could be an important element in the epigenetic development of an individual either a human or a robot to modulate the learning during life long learning.

\section{REFERENCES}

[1] Gregor Schöner. Dynamical Systems Approaches to Cognition, pages 101-126. Cambridge Handbooks in Psychology. Cambridge University Press, 2008.

[2] Strogatz and Ian Stewart. Coupled oscillators and biological synchronization. Scientific American, 1993.

[3] Issartel, Marin, and Cadopi. Unintended interpersonal co-ordination: "can we march to the beat of our own drum?". Neurosci Lett. 2007 Jan 16;411(3):174-, 2007.

[4] Bernieri and Rosenthal. Fundamentals of non verbal behaviour, p 401-432. R.S Feldman and B. Rime, 1991.

[5] Michael J. Richardson, Kerry L. Marsh, Robert W. Isenhower, Justin R.L. Goodman, and R.C. Schmidt. Rocking together: Dynamics of intentional and unintentional interpersonal coordination. Human Movement Science, 26(6):867 - 891, 2007.

[6] Paula Fiztpatrick, Teresa Mitchell, Jean Frazier, and Richard Schmidt. Exploring the behavioral neural processes underlying social synchronization of inidviduals with and without social deficits, Studies in perception and actions XIII, p40-44. Julie A. Weast-Knapp and MaryLauren, 2015.

[7] Richardson, Rick, Dale, and Kirkhal. the art of conversation is coordination. Apsychological science vol 18 number 5, 2007.

[8] FrankJ. Bernieri. Coordinated movement and rapport in teacher-student interactions. Journal of Nonverbal Behavior, 12(2):120-138, 1988.

[9] Chloë Leclère ans Sylvie Viaux, Marie Avril, Catherine Achar, Mohamed Chetouani, Sylvain Missonnier, and David Cohen. Why synchrony matters during mother-child interactions: A systematic review. Ed. Sharon Dekel. PLoS ONE 9.12 (2014): e113571. PMC. Web. 9 Sept. 2015., 2014.

[10] H. Ogawa and T. Watanabe. Interrobot: a speech driven embodied interaction robot. pages $322-327$, 2000.

[11] Daisuke Sakamoto, Takayuki Kanda, Tetsuo Ono, Masayuki Kamashima, Michita Imai, and Hiroshi Ishiguro. Cooperative embodied communication emerged by interactive humanoid robots. International Journal of Human-Computer Studies, 62(2):247 $-265,2005$. Subtle expressivity for characters and robots.

[12] Kyongsik Yun, Kastumo Watanabe, and Shinsuke Shimojo. Interpersonal body and neural synchronization as a marker of implicit social interation. Scientific Reports - 2:959 -, 2012.
[13] S.K. Hasnain, P. Gaussier, and G. Mostafaoui. Synchrony as a tool to establish focus of attention for autonomous robots. In Intelligent Robots and Systems (IROS), 2012 IEEE/RSJ International Conference on, pages 2423-2428, 2012.

[14] T. Lorenz, A. Mörtl, and S. Hirche. Movement synchronization fails during non-adaptive human-robot interaction. In 2013 8th ACM/IEEE International Conference on Human-Robot Interaction (HRI), pages 189-190, March 2013.

[15] Shinya Fujii, Masaya Hirashima, Kazutoshi Kudo, Tatsuyuki Ohtsuki, Yoshihiko Nakamura, and Shingo Oda. Synchronization error of drum kit playing with a metronome at different tempi by professional drummers. Music Perception: An Interdisciplinary Journal, 28(5):491-503, 2011.

[16] Joanne Lumsden, Lynden K. Miles, and C. Neil Macrae. Sync or sink? interpersonal synchrony impacts self-esteem. Frontiers in Psychology, 5:1064, 2014.

[17] Pierre Andry, Philippe Gaussier, Sorin Moga, JeanPaul Banquet, and Jacqueline Nadel. Learning and communication via imitation: An autonomous robot perspective. IEEE Transactions on Systems, Man, and Cybernetics-Part A: Systems and Humans, 31(5):431$442,2001$.

[18] Jacqueline Nadel, Arnaud Revel, Pierre Andry, and Philippe Gaussier. Toward communication: first imitations in infants, low-functioning children with autism and robots. Interaction Studies, 5(1):45-74, 2004.

[19] Pierre Andry, Arnaud Blanchard, and Philippe Gaussier. Using the rhythm of nonverbal humanrobot interaction as a signal for learning. IEEE Transactions on Autonomous Mental Development, 3(1):3042, 2011.

[20] James Pataleone. Synchronization of metronomes. American Journal of Physics, 2002.

[21] J. A. Scott Kelso. Haken-Kelso-Bunz model. Scholarpedia, 3(10):1612, 2008. revision 91336.

[22] H.R Wilson and J.D Cowan J. Excitatory and inhibitory interactions in localized populations of model neurons. Biophys. J., Vol. 12, 1972.

[23] Syed Khursheed Hasnain, Ghiles Mostafaoui, and Philippe Gaussier. A synchrony-based perspective for partner selection and attentional mechanism in human-robot interaction. Paladyn Journal of Behavioral Robotics, 3(3):156-171, 2012.

[24] Ken Prepin and Philippe Gaussier. How an Agent Can Detect and Use Synchrony Parameter of Its Own Interaction with a Human?, pages 50-65. Springer Berlin Heidelberg, 2010.

[25] Tomer Amiaz, Eyal Lubetzky, and Nahum Kiryati. Coarse to over-fine optical flow estimation. Pattern Recogn., 40(9):2496-2503, September 2007.

[26] E. Ansermin, G. Mostafaoui, X. Sargentini, and P. Gaussier. Unintentional entrainment effect in a context of human robot interaction an experimental study. 2000. 